\title{
PSEUDO ALMOST PERIODIC SOLUTIONS OF AN ITERATIVE EQUATION WITH VARIABLE COEFFICIENTS
}

\author{
HOU YU ZHAO AND MICHAL FEČKAN
}

Received 09 June, 2016

\begin{abstract}
In this paper, the exponential dichotomy, and Tikhonov and Banach fixed point theorems are used to study the existence and uniqueness of pseudo almost periodic solutions of nonhomogeneous iterative functional differential equations of the form $x^{\prime}(t)=\lambda_{1}(t) x(t)+$ $\lambda_{2}(t) x^{[2]}(t)+\ldots+\lambda_{n}(t) x^{[n]}(t)+f(t)$.

2010 Mathematics Subject Classification: 39B12; 39B82
\end{abstract}

Keywords: iterative functional differential equation, pseudo almost periodic solutions, fixed point theorem

\section{INTRODUCTION}

Recently, iterative functional differential equations of the form

$$
x^{\prime}(t)=H\left(x^{[0]}(t), x^{[1]}(t), x^{[2]}(t), \ldots, x^{[n]}(t)\right)
$$

have appeared in several papers, where $x^{[0]}(t)=t, x^{[i]}(t)=x\left(x^{[i-1]}(t)\right), i \geq 1$. In [4], Cooke points out that it is highly desirable to establish the existence and stability properties of periodic solutions for equations of the form

$$
x^{\prime}(t)+a x(t-h(t, x(t)))=F(t)
$$

in which the lag $h(t, x(t))$ implicitly involves $x(t)$. Stephan [14] studies the existence of periodic solutions of equation

$$
x^{\prime}(t)+a x(t-r+\mu h(t, x(t)))=F(t) .
$$

Eder [6] considers the iterative functional differential equation

$$
x^{\prime}(t)=x^{[2]}(t)
$$

This work was partially supported by the National Natural Science Foundation of China (Grant No. 11326120, 11501069), Foundation of Chongqing Municipal Education Commission (Grant No. KJ1400528), the Grants VEGA-MS 1/0071/14, VEGA-SAV 2/0153/16 and by the Slovak Research and Development Agency under the contract No. APVV-14-0378. 
and obtains that every solution either vanishes identically or is strictly monotonic. Fečkan [7] studies the equation

$$
x^{\prime}(t)=f\left(x^{[2]}(t)\right)
$$

by obtaining an existence theorem for solutions satisfying $x(0)=0$. Later, Wang and Si [17] study

$$
x^{\prime}\left(x^{[r]}(t)\right)=c_{0} z+c_{1} x(t)+c_{2} x^{[2]}(t)+\ldots+x^{[n]}(t),
$$

and show the existence theorem of analytic solutions. In particularly, Si and Cheng [12] discusses the smooth solutions of equation of

$$
x^{\prime}(t)=\lambda_{1} x(t)+\lambda_{2} x^{[2]}(t)+\ldots+\lambda_{n} x^{[n]}(t)+f(t) .
$$

For some various properties of solutions for several iterative functional differential equations, we refer the interested reader to $[10,11,13,15]$.

On the other hand, the existence of pseudo almost periodic solutions is among the most attractive topics in qualitative theory of differential equations due to their applications, especially in biology, economics and physics $[1,3,5,18,20]$. As pointed out by Ait Das and Ezzinbi [1], it is an interesting thing to study the pseudo almost periodic systems with delays. It is obviously that iterative functional differential equations are special type state-dependent delay differential equations. In [9], Liu pointed that the properties of the almost periodic functions do not always hold in the set of pseudo almost periodic functions and given an example: when $x(t)$ is a pseudo almost periodic function, $x(x(t))$ may not be a pseudo almost periodic function. To the best of our knowledge, there are few results about pseudo almost periodic solutions for iterative functional differential equations except [9] and [16].

In the present work, we propose an existence result for pseudo almost periodic solutions of iterative functional differential equations

$$
x^{\prime}(t)=\lambda_{1}(t) x(t)+\lambda_{2}(t) x^{[2]}(t)+\ldots+\lambda_{n}(t) x^{[n]}(t)+f(t) .
$$

by using Tikhonov fixed theorem. Uniqueness of the solution is achieved by Banach fixed point theorem.

This paper is organized as follows. In Section 2 we give two theorems which are used in Section 3 to establish the main existence result. In Section 4, we show that (1.1) has a unique pseudo almost periodic solution under some suitable conditions and present an example to illustrate the theory. Related problems are also studied in [2]. 


\section{PRELIMINARIES}

Let $C(\mathbb{R}, \mathbb{R})$ denote the set of all continuous functions from $\mathbb{R}$ to $\mathbb{R}$ endowed with the usual metric

$$
d(f, g)=\sum_{m=1}^{\infty} 2^{-m} \frac{\|f-g\|_{m}}{1+\|f-g\|_{m}} \quad \text { for } \quad\|f-g\|_{m}=\max _{t \in[-m, m]}|f(t)-g(t)|,
$$

so the topology on $C(\mathbb{R}, \mathbb{R})$ is the uniform convergence on each compact intervals of $\mathbb{R}$. We also consider the set $B C(\mathbb{R}, \mathbb{R})$ of all bounded and continuous functions from $\mathbb{R}$ to $\mathbb{R}$ with the norm $\|f\|=\sup _{t \in \mathbb{R}}|f(t)|$, so the topology on $B C(\mathbb{R}, \mathbb{R})$ is the uniform convergence on $\mathbb{R}$. We denote by $A P(\mathbb{R}, \mathbb{R})$ the set of all almost periodic functions from $\mathbb{R}$ to $\mathbb{R}$. Define the set

$$
P A P_{0}(\mathbb{R}, \mathbb{R})=\left\{g \in B C(\mathbb{R}, \mathbb{R})\left|\lim _{T \rightarrow+\infty} \frac{1}{2 T} \int_{-T}^{T}\right| g(t) \mid d t=0\right\} .
$$

A function $\varphi \in B C(\mathbb{R}, \mathbb{R})$ is called pseudo almost periodic if it can be expressed as $\varphi=h+g$, where $h \in A P(\mathbb{R}, \mathbb{R})$ and $g \in P A P_{0}(\mathbb{R}, \mathbb{R})$. The collection of such functions will be denoted by $P A P(\mathbb{R}, \mathbb{R})$. In particular, $(P A P(\mathbb{R}, \mathbb{R}),\|\cdot\|)$ is a Banach space [19]. For $M, L>0$, define

$$
\begin{gathered}
B_{C}(M, L)=\left\{\varphi \in C(\mathbb{R}, \mathbb{R})|| \varphi(t)|\leq M,| \varphi\left(t_{2}\right)-\varphi\left(t_{1}\right)|\leq L| t_{2}-t_{1} \mid,\right. \\
\left.\quad \text { for all } t, t_{1}, t_{2} \in \mathbb{R}\right\}, \\
B_{S}(M, L)=S(\mathbb{R}, \mathbb{R}) \cap B_{C}(M, L), \quad S \in\left\{P A P, A P, P A P_{0}\right\} .
\end{gathered}
$$

From [9], it is easy to see that $B_{S}(M, L), S \in\left\{P A P, A P, P A P_{0}, C\right\}$ are closed convex and bounded subsets of $B C(\mathbb{R}, \mathbb{R})$ and $C(\mathbb{R}, \mathbb{R})$, respectively. Furthermore, by the Arzelá-Ascoli theorem, the subset $B_{C}(M, L)$ is compact in $C(\mathbb{R}, \mathbb{R})$.

On the other hand, the subset $B_{C}(M, L)$ is not precompact in $B C(\mathbb{R}, \mathbb{R})$, since a sequence $\left\{M \operatorname{sech} \frac{L(t+n)}{M}\right\}_{n=1}^{\infty} \in B_{C}(M, L)$ has no a convergent subsequence in $B C(\mathbb{R}, \mathbb{R})$.

Furthermore, the subset $B_{A P}(M, L)$ is not compact in $C(\mathbb{R}, \mathbb{R})$, since we consider the function from [8]

$$
g(t)=\sum_{k=1}^{\infty} \frac{1}{k} \sin ^{2} \frac{t}{2^{k}}
$$

From $\frac{1}{k} \sin ^{2} \frac{t}{2^{k}} \leq \frac{1}{k} \frac{t^{2}}{4^{k}}$ and that the series $\sum_{k=1}^{\infty} \frac{1}{k} \frac{t^{2}}{4^{k}}$ convergents, we see that

$$
g_{n}(t)=\sum_{k=1}^{n} \frac{1}{k} \sin ^{2} \frac{t}{2^{k}} \rightarrow g(t)
$$


in $C(\mathbb{R}, \mathbb{R})$. Similarly we derive

$$
g_{n}^{\prime}(t)=\sum_{k=1}^{n} \frac{1}{k 2^{k-1}} \sin \frac{t}{2^{k}} \cos \frac{t}{2^{k}} \rightarrow g^{\prime}(t)
$$

in $B C(\mathbb{R}, \mathbb{R})$ and $\left\|g_{n}^{\prime}\right\| \leq 2,\left\|g^{\prime}\right\| \leq 2$. Take

$$
\varphi_{n}(t)=\min \left\{1, g_{n}(t)\right\}=\frac{1+g_{n}(t)-\left|1-g_{n}(t)\right|}{2} .
$$

Clearly $\varphi_{n} \in B_{A P}(1,2)$ and $\varphi_{n} \rightarrow \varphi=\min \{1, g\}$ in $C(\mathbb{R}, \mathbb{R})$. Next we know from [8] that $\limsup \sup _{t \rightarrow \infty} g(t)=\infty$ and $\liminf _{t \rightarrow \infty} g(t)=0$. So applying [8, Lemma 2.1], we know that $\varphi \notin A P(\mathbb{R}, \mathbb{R})$. Hence the subset $B_{A P}(1,2)$ is not complete in $C(\mathbb{R}, \mathbb{R})$. By taking the sequence $\left\{\varphi_{n}\left(\frac{L}{2 M} t\right)\right\}_{n=1}^{\infty}$, we see that $B_{A P}(M, L)$ is not complete in $C(\mathbb{R}, \mathbb{R})$ for any $M>0$ and $L>0$. Since $B_{A P}(M, L) \subset B_{C}(M, L), B_{A P}(M, L)$ is just precompact in $C(\mathbb{R}, \mathbb{R})$. We have also shown that $B_{A P}(M, L)$ are not precompact in $B C(\mathbb{R}, \mathbb{R})$, but they are complete.

Furthermore, for any $k \in \mathbb{N}$, we have $\frac{1}{2^{k} \pi} \int_{0}^{2^{k} \pi} \varphi(t) d t \geq \frac{1}{2^{k} \pi} \int_{0}^{2^{k} \pi} \sin ^{2} \frac{t}{2}=\frac{1}{2}$, so $\varphi \notin P A P_{0}(\mathbb{R}, \mathbb{R})$. But we do not know $\varphi \notin P A P(\mathbb{R}, \mathbb{R})$.

Finally, taking

$$
\psi_{n}(t)=\left\{\begin{array}{cl}
0 & \text { for }|t| \geq n+1, \\
1 & \text { for }|t| \leq n, \\
t+n+1 & \text { for } t \mid \in[-n-1,-n], \\
n+1-t & \text { for } t \leq[n, n+1],
\end{array}\right.
$$

we see that $\psi_{n} \in B_{P A P_{0}}(1,1)$ and $\psi_{n} \rightarrow 1$ in $C(\mathbb{R}, \mathbb{R})$, so $B_{P A P_{0}}(M, L)$ are not complete in $C(\mathbb{R}, \mathbb{R})$. We have also shown that $B_{P A P_{0}}(M, L)$ are not precompact in $B C(\mathbb{R}, \mathbb{R})$, but they are complete.

Summarizing, we arrive at the following result.

Theorem 1. The subsets $B_{S}(M, L), S \in\left\{P A P, A P, P A P_{0}, C\right\}$ are not precompact in $B C(\mathbb{R}, \mathbb{R})$. They are precompact in $C(\mathbb{R}, \mathbb{R})$. Furthermore, $B_{A P}(M, L)$ and $B_{P A P_{0}}(M, L)$ are just precompact in $C(\mathbb{R}, \mathbb{R})$, while $B_{C}(M, L)$ is compact in $C(\mathbb{R}, \mathbb{R})$.

So (non-)compactness of $B_{P A P}(M, L)$ in $C(R, R)$ is still open.

Finally, we recall Tikhonov fixed point theorem.

Theorem 2. (Tikhonov) Let $\Omega$ be a non-empty compact convex subset of a locally convex topological vector space $X$. Then any continuous function $A: \Omega \rightarrow \Omega$ has a fixed point. 


\section{EXISTENCE RESULT}

In this section, the existence of pseudo almost periodic solutions of equation (1.1) will be studied. Throughout this paper, it will be assumed that $\lambda_{1}: \mathbb{R} \rightarrow(-\infty, 0)$ is an almost periodic function, $\lambda_{i}: \mathbb{R} \rightarrow \mathbb{R}$ are pseudo almost periodic functions, and

$$
\lambda_{1}^{+}=\sup _{t \in \mathbb{R}} \lambda_{1}(t)<0, \lambda_{i}^{+}=\sup _{t \in \mathbb{R}}\left|\lambda_{i}(t)\right|,
$$

where $i=2, \ldots, n$.

Now we apply Theorem 2 . Let $X$ be either $P A P(\mathbb{R}, \mathbb{R})$ or $B C(\mathbb{R}, \mathbb{R})$ but fixed. If $\varphi \in X$, from Corollary 5.4 in [19], it follows $\varphi^{[2]} \in X$. Consider an auxiliary equation

$$
x^{\prime}(t)=\lambda_{1}(t) x(t)+\lambda_{2}(t) \varphi^{[2]}(t)+\ldots+\lambda_{n}(t) \varphi^{[n]}(t)+f(t),
$$

where $f \in P A P(\mathbb{R}, \mathbb{R})$ and $\lambda_{1}(t)<0$. It is easy to see that the linear equation

$$
x^{\prime}(t)=\lambda_{1}(t) x(t)
$$

admits an exponential dichotomy on $\mathbb{R}$, by Theorem 2.3 in [18], we know that (3.1) has exactly one solution

$$
x_{\varphi}(t)=\int_{-\infty}^{t} e^{\int_{s}^{t} \lambda_{1}(u) d u}\left(\sum_{i=2}^{n} \lambda_{i}(s) \varphi^{[i]}(s)+f(s)\right) d s
$$

in $X$.

Now take $f \in B_{P A P}\left(M^{\prime}, L^{\prime}\right)$ for constants $M^{\prime}$ and $L^{\prime}$. Let $A: B_{C}(M, L) \rightarrow$ $B C(\mathbb{R}, \mathbb{R})$ be defined by

$$
(A \varphi)(t)=\int_{-\infty}^{t} e^{\int_{s}^{t} \lambda_{1}(u) d u}\left(\sum_{i=2}^{n} \lambda_{i}(s) \varphi^{[i]}(s)+f(s)\right) d s, t \in \mathbb{R} .
$$

Note $A: B_{P A P}(M, L) \rightarrow P A P(\mathbb{R}, \mathbb{R})$.

Lemma 1. For any $\varphi, \psi \in B_{C}(M, L), t_{1}, t_{2} \in \mathbb{R}$, the following inequality hold,

$$
\left\|\varphi^{[n]}-\psi^{[n]}\right\| \leq \sum_{j=0}^{n-1} L^{j}\|\varphi-\psi\|, n=1,2, \ldots
$$

Proof. It can be obtained by direct calculation by the definition of $B_{C}(M, L)$.

Lemma 2. Operator $A: B_{C}(M, L) \rightarrow C(\mathbb{R}, \mathbb{R})$ is continuous.

Proof. Let $\varphi_{j} \rightarrow \varphi_{0}$ as $j \rightarrow \infty$ for $\varphi_{j} \in B_{C}(M, L), j \in \mathbb{N}_{0}=\mathbb{N} \cup\{0\}$ uniformly on any compact interval $[-m, m], m \in \mathbb{N}$ of $\mathbb{R}$. Set

$$
h_{j}(s)=\sum_{i=2}^{n} \lambda_{i}(s) \varphi_{j}^{[i]}(s)+f(s), \quad j \in \mathbb{N}_{0} .
$$


Then $h_{j} \rightarrow h_{0}$ uniformly on $[-m, m]$. Next we have

$$
\left|e^{\int_{s}^{-m} \lambda_{1}(u) d u} h_{j}(s)\right| \leq\left(M \sum_{i=2}^{n} \lambda_{i}^{+}+\|f\|\right) e^{-\lambda_{1}^{+}(s+m)}, \quad s \in[-\infty,-m] .
$$

Since

$$
\int_{-\infty}^{-m} e^{-\lambda_{1}^{+}(s+m)} d s=-\frac{1}{\lambda_{1}^{+}}<\infty
$$

we can apply the Lebesgue dominated convergence theorem to obtain $\left(A \varphi_{j}\right)(-m) \rightarrow$ $\left(A \varphi_{0}\right)(-m)$. From (3.1),

$$
x_{j}^{\prime}(t)-x_{0}^{\prime}(t)=\lambda_{1}(t)\left(x_{j}(t)-x_{0}(t)\right)+h_{j}(t)-h_{0}(t)
$$

for $x_{j}(t)=\left(A \varphi_{j}\right)(t), k \in \mathbb{N}_{0}$. Integrating the both sides of (3.4) from $-m$ to $t$, we have

$$
\begin{gathered}
x_{j}(t)-x_{0}(t)=x_{j}(-m)-x_{0}(-m)+\int_{-m}^{t} \lambda_{1}(s)\left(x_{j}(s)-x_{0}(s)\right) d s \\
+\int_{-m}^{t}\left(h_{j}(s)-h_{0}(s)\right) d s
\end{gathered}
$$

and

$\left|x_{j}(t)-x_{0}(t)\right| \leq\left|x_{j}(-m)-x_{0}(-m)\right|+2 m\left\|h_{j}-h_{0}\right\|_{m}-\lambda_{1}^{+} \int_{-m}^{t}\left|x_{j}(s)-x_{0}(s)\right| d s$ for any $t \in[-m, m]$. Then Gronwall's inequality implies

$$
\left\|x_{j}-x_{0}\right\|_{m} \leq\left(\left|x_{j}(-m)-x_{0}(-m)\right|+2 m\left\|h_{j}-h_{0}\right\|_{m}\right) e^{-2 \lambda_{1}^{+} m},
$$

which means

$$
\left\|A \varphi_{j}-A \varphi_{0}\right\|_{m} \leq\left(\left|A \varphi_{j}(-m)-A \varphi_{0}(-m)\right|+2 m\left\|h_{j}-h_{0}\right\|_{m}\right) e^{-2 \lambda_{1}^{+} m} .
$$

Hence $A \varphi_{j}(t) \rightarrow A \varphi_{0}(t)$ uniformly on $t \in[-m, m]$. Since $m \in \mathbb{N}$ is arbitrarily, we get $A \varphi_{j} \rightarrow A \varphi_{0}$ in $C(\mathbb{R}, \mathbb{R})$, i.e., $A: B_{C}(M, L) \rightarrow C(\mathbb{R}, \mathbb{R})$ is continuous. This proves the continuity of $A$.

Of course, then $A: B_{P A P}(M, L) \rightarrow C(\mathbb{R}, \mathbb{R})$ is continuous as well.

Theorem 3. Suppose

$$
-\lambda_{1}^{+}-\sum_{i=2}^{n} \lambda_{i}^{+}>0
$$

Then for any $f \in B_{P A P}\left(M^{\prime}, L^{\prime}\right), E q$. (1.1) has a solution

$$
\varphi \in \overline{B_{P A P}(M, L)} \subset C(\mathbb{R}, \mathbb{R})
$$


for the constants $M$ and L given by

$$
M=-\frac{M^{\prime}}{\lambda_{1}^{+}+\sum_{i=2}^{n} \lambda_{i}^{+}}, \quad L=\frac{2 M^{\prime} \lambda_{1}^{+}}{\lambda_{1}^{+}+\sum_{i=2}^{n} \lambda_{i}^{+}} .
$$

Proof. For any $\varphi, \psi \in B_{C}(M, L), t, t_{1}, t_{2} \in \mathbb{R}$, from (3.6) we have

$$
\begin{gathered}
|(A \varphi)(t)| \leq \sum_{i=2}^{n} \lambda_{i}^{+}\left|\int_{-\infty}^{t} e^{\int_{s}^{t} \lambda_{1}(u) d u} \varphi^{[i]}(s) d s\right|+\left|\int_{-\infty}^{t} e^{\int_{s}^{t} \lambda_{1}(u) d u} f(s) d s\right| \\
\leq-\frac{M}{\lambda_{1}^{+}} \sum_{i=2}^{n} \lambda_{i}^{+}-\frac{M^{\prime}}{\lambda_{1}^{+}}=M .
\end{gathered}
$$

Without loss of generality, assume $t_{2} \geq t_{1}$, then

$$
\begin{gathered}
\left|(A \varphi)\left(t_{2}\right)-(A \psi)\left(t_{1}\right)\right| \\
\leq \sum_{i=2}^{n} \lambda_{i}^{+}\left|\int_{-\infty}^{t_{2}} e^{\int_{s}^{t_{2}} \lambda_{1}(u) d u} \varphi^{[i]}(s) d s-\int_{-\infty}^{t_{1}} e^{\int_{s}^{t_{1}} \lambda_{1}(u) d u} \varphi^{[i]}(s) d s\right| \\
+\left|\int_{-\infty}^{t_{2}} e^{\int_{s}^{t_{2}} \lambda_{1}(u) d u} f(s) d s-\int_{-\infty}^{t_{1}} e^{\int_{s}^{t_{1}} \lambda_{1}(u) d u} f(s) d s\right| \\
\leq \sum_{i=2}^{n} \lambda_{i}^{+}\left[\left|\int_{-\infty}^{t_{1}} e^{\int_{s}^{t_{1}} \lambda_{1}(u) d u}\left(e^{\int_{t_{1}}^{t_{2}} \lambda_{1}(u) d u}-1\right) \varphi^{[i]}(s) d s\right|\right. \\
+\left[\left|\int_{t_{1}}^{t_{2}} e^{\int_{s}^{t_{2}} \lambda_{1}(u) d u} \varphi^{[i]}(s) d s\right|\right] \\
\left.e^{\int_{s}^{t_{1}} \lambda_{1}(u) d u}\left(e^{\int_{t_{1}}^{t_{2}} \lambda_{1}(u) d u}-1\right) f(s) d s|+| \int_{t_{1}}^{t_{2}} e^{f_{s}^{t_{2}} \lambda_{1}(u) d u} f(s) d s \mid\right] \\
\leq M \sum_{i=2}^{n} \lambda_{i}^{+}\left[\left|\lambda_{1}^{+}\right|\left|t_{2}-t_{1}\right|\left|\int_{-\infty}^{t_{1}} e^{\lambda_{1}^{+}\left(t_{1}-s\right)} d s\right|+\left|\int_{t_{1}}^{t_{2}} e^{\lambda_{1}^{+}\left(t_{2}-s\right)} d s\right|\right] \\
+M^{\prime}\left[\left|\lambda_{1}^{+}\right|\left|t_{2}-t_{1}\right|\left|\int_{-\infty}^{t_{1}} e^{\lambda_{1}^{+}\left(t_{1}-s\right)} d s\right|+\left|\int_{t_{1}}^{t_{2}} e^{\lambda_{1}^{+}\left(t_{2}-s\right)} d s\right|\right] \\
\leq 2\left(M \sum_{i=2}^{n} \lambda_{i}^{+}+M^{\prime}\right)\left|t_{2}-t_{1}\right|=L\left|t_{2}-t_{1}\right| .
\end{gathered}
$$

This shows that $A \varphi \in B_{C}(M, L)$. Hence $A: B_{C}(M, L) \rightarrow B_{C}(M, L)$ and also $A$ : $B_{P A P}(M, L) \rightarrow B_{P A P}(M, L)$. By Lemma 2 we get $A: \overline{B_{P A P}(M, L)} \rightarrow \overline{B_{P A P}(M, L)}$. So all conditions of Tikhonov fixed theorem are satisfied for $A$ with $\Omega=\overline{B_{P A P}(M, L)}$ and $X=C(\mathbb{R}, \mathbb{R})$. Thus there exists a fixed point $\varphi$ in $\overline{B_{P A P}(M, L)}$ of $A$. This is 
equivalent to say that $\varphi$ is a solution of (1.1) in $\overline{B_{P A P}(M, L)}$. This completes the proof.

The solution $\varphi$ of Theorem 3 is a limit in $C(\mathbb{R}, \mathbb{R})$ of a sequence of pseudo almost periodic functions in $B_{P A P}(M, L)$, i.e., $\varphi$ is a uniform approximation on each compact intervals of $\mathbb{R}$ of a sequence of pseudo almost periodic functions. If $f \in$ $B_{A P}\left(M^{\prime}, L^{\prime}\right)$ then we know by Theorem 1 only that in general, the solution $\varphi$ of Theorem 3 belongs just to $f \in \overline{B_{A P}(M, L)}$.

\section{UNIQUENESS RESULT}

In this section, uniqueness of (1.1) will be proved, moreover, an example will be showed.

Theorem 4. Suppose (3.5). Then for any $f \in B_{P A P}\left(M^{\prime}, L^{\prime}\right)$, Eq. (1.1) has the unique solution $\varphi \in B_{P A P}(M, L)$ provided that it holds

$$
\Gamma=-\frac{1}{\lambda_{1}^{+}} \sum_{i=2}^{n} \sum_{j=0}^{i-1} \lambda_{i}^{+} L^{j}<1
$$

for the constants $M$ and $L$ given by (3.6).

Proof. We already known that $A: B_{P A P}(M, L) \rightarrow B_{P A P}(M, L)$ where we consider $B_{P A P}(M, L)$ in $B C(\mathbb{R}, \mathbb{R})$. For $\varphi, \psi \in B_{P A P}(M, L)$, by (3.2) and (3.3)

$$
\begin{gathered}
|\varphi(t)-\psi(t)|=|(A \varphi)(t)-(A \psi)(t)| \leq \sum_{i=2}^{n} \lambda_{i}^{+} \int_{-\infty}^{t} e^{\int_{s}^{t}} \lambda_{1}(u) d u\left|\varphi^{[i]}(s)-\psi^{[i]}(s)\right| d s \\
\leq-\frac{1}{\lambda_{1}^{+}} \sum_{i=2}^{n} \sum_{j=0}^{i-1} \lambda_{i}^{+} L^{j}\|\varphi-\psi\|=\Gamma\|\varphi-\psi\|,
\end{gathered}
$$

thus

$$
\|\varphi-\psi\| \leq \Gamma\|\varphi-\psi\|
$$

From (4.1), we know $\Gamma<1$ and $B_{P A P}(M, L)$ is closed in the Banach space $B C(\mathbb{R}, \mathbb{R})$. The Banach fixed point theorem gives a unique fixed point of $A$ in $B_{P A P}(M, L)$. The proof is finished.

Example 1. Now, we apply Theorem 4. Consider the following equation:

$$
\begin{aligned}
x^{\prime}(t)= & -(|\sin t+\sin \sqrt{3} t|+6) x(t)+\frac{1}{5}(|\cos t+\cos \sqrt{5} t|+8) x(x(t)) \\
& +\frac{1}{7}(\sin t+\sin \sqrt{2} t+\operatorname{sech} t)
\end{aligned}
$$

where $\lambda_{1}(t)=-(|\sin t+\sin \sqrt{3} t|+6), \lambda_{2}(t)=\frac{1}{5}(|\cos t+\cos \sqrt{5} t|+8)$ and $f(t)=$ $\frac{1}{7}(\sin t+\sin \sqrt{2} t+\operatorname{sech} t)$. Take $M^{\prime}=\frac{3}{7}$ and $L^{\prime}=\frac{4}{7}$. A simple calculation yields 
$\lambda_{1}^{+}=-6$ and $\lambda_{2}^{+}=2$, so (3.5) holds: $-\lambda_{1}^{+}-\lambda_{2}^{+}=4>0$. Then by (3.6) we derive $M=\frac{3}{28}$ and $L=\frac{9}{7}$. Thus (4.1) gives $\Gamma=\frac{16}{21}<1$. Hence by Theorem 4, Eq. (4.2) has a unique solution in $B_{P A P}\left(\frac{3}{28}, \frac{9}{7}\right)$.

\section{REFERENCES}

[1] E. Ait Dads and K. Ezzinbi, "Pseudo almost periodic solutions of some delay differential equations.” J. Math. Anal. Appl., vol. 201, no. 3, pp. 840-850, art. no. 0287, 1996, doi: 10.1006/jmaa.1996.0287.

[2] J. Andres and A. M. Bersani, "Almost-periodicity problem as a fixed-point problem for evolution inclusions." Topol. Methods Nonlinear Anal., vol. 18, no. 2, pp. 337-349, 2001.

[3] F. Chérif, "Existence and global exponential stability of pseudo almost periodic solution for SICNNs with mixed delays." J. Appl. Math. Comput., vol. 39, no. 1-2, pp. 235-251, 2012, doi: 10.1007/s12190-011-0520-1.

[4] K. Cooke, "Functional-differential equations: Some models and perturbation problems." Differ. Equations dynam. Systems, Proc. Int. Sympos. Puerto Rico 1965, 167-183 (1967)., 1967.

[5] L. Duan and L. Huang, "Pseudo almost periodic dynamics of delay Nicholson's blowflies model with a linear harvesting term." Math. Methods Appl. Sci., vol. 38, no. 6, pp. 1178-1189, 2015, doi: 10.1002/mma.3138.

[6] E. Eder, "The functional differential equation $x^{\prime}(t)=x(x(t))$." J. Differ. Equations, vol. 54, pp. 390-400, 1984, doi: 10.1016/0022-0396(84)90150-5.

[7] M. Fečkan, "On a certain type of functional differential equations." Math. Slovaca, vol. 43, no. 1, pp. 39-43, 1993.

[8] A. Haraux and P. Souplet, "An example of uniformly recurrent function which is not almost periodic.” J. Fourier Anal. Appl., vol. 10, no. 2, pp. 217-220, 2004, doi: 10.1007/s00041-004-8012-4.

[9] B. Liu and C. Tunç, "Pseudo almost periodic solutions for a class of first order differential iterative equations." Appl. Math. Lett., vol. 40, pp. 29-34, 2015, doi: 10.1016/j.aml.2014.08.019.

[10] S. Minsker, "On splitting the area under curves." J. Differ. Equations, vol. 26, pp. 443-457, 1977, doi: 10.1016/0022-0396(77)90090-0.

[11] S. Minsker, "On splitting the area under curves. II.” J. Differ. Equations, vol. 45, pp. 182-190, 1982, doi: 10.1016/0022-0396(82)90064-X.

[12] J. G. Si and S. S. Cheng, "Smooth solutions of a nonhomogeneous iterative functional-differential equation.” Proc. R. Soc. Edinb., Sect. A, Math., vol. 128, no. 4, pp. 821-831, 1998, doi: 10.1017/S0308210500021806.

[13] S. Staněk, "On global properties of solutions of functional differential equation $x^{\prime}(t)=x(x(t))+$ $x(t) . "$ Dyn. Syst. Appl., vol. 4, no. 2, pp. 263-278, 1995.

[14] B. H. Stephan, "On the existence of periodic solutions of $z^{\prime}(t)=-a z(t-r+u k(t, z(t)))+F(t)$." J. Differ. Equations, vol. 6, pp. 408-419, 1969, doi: 10.1016/0022-0396(69)90002-3.

[15] K. Wang, "On the equation $x^{\prime}(t)=f(x(x(t)))$." Funkc. Ekvacioj, Ser. Int., vol. 33, no. 3, pp. 405-425, 1990.

[16] W. Wang, "Positive pseudo almost periodic solutions for a class of differential iterative equations with biological background." Appl. Math. Lett., vol. 46, pp. 106-110, 2015, doi: 10.1016/j.aml.2015.02.015.

[17] X. P. Wang and J. G. Si, "Analytic solutions of an iterative functional differential equation." $J$. Math. Anal. Appl., vol. 262, no. 2, pp. 490-498, 2001, doi: 10.1006/jmaa.2001.7527.

[18] C. Zhang, "Pseudo almost periodic solutions of some differential equations. II." J. Math. Anal. Appl., vol. 192, no. 2, pp. 543-561, 1995, doi: 10.1006/jmaa.1995.1189.

[19] C. Zhang, Almost periodic type functions and ergodicity. Dordrecht: Kluwer Academic Publishers; Beijing: Science Press, 2003. 
[20] H. Zhang, "New results on the positive pseudo almost periodic solutions for a generalized model of hematopoiesis." Electron. J. Qual. Theory Differ. Equ., vol. 2014, p. 10, 2014.

Authors' addresses

Hou Yu Zhao

School of Mathematics, Chongqing Normal University, Chongqing 401331, P.R.China

Department of Pure Mathematics, University of Waterloo, Waterloo, ON N2L 3G1, Canada E-mail address: houyu19@gmail.com

Michal Fečkan

Department of Mathematical Analysis and Numerical Mathematics, Comenius University in Bratislava, Mlynská dolina, 84248 Bratislava, Slovakia

Mathematical Institute of Slovak Academy of Sciences, Štefánikova 49, 81473 Bratislava, Slovakia

E-mail address: Michal.Feckan@fmph.uniba.sk 